ARCHIVO ESPAÑOL DE ARTE, LXXXVIII, 351

JULIO-SEPTIEMBRE 2015, pp. 310-314

ISSN: 0004-0428, eISSN: 1988-8511

doi: $10.3989 /$ aearte. 2015.20

\title{
DOS NUEVOS DIBUJOS DE CLAUDIO COELLO \\ EN LA BIBLIOTECA NACIONAL DE ESPAÑA
}

\author{
Teresa Zapata Fernández de la Hoz y Juan Carlos Gómez Aragüete
}

Se atribuyen al pintor madrileño Claudio Coello (1642-1693) dos dibujos de tema similar conservados en la Biblioteca Nacional de España, considerados hasta ahora como anónimos.

Palabras clave: Claudio Coello; Dibujos; Biblioteca Nacional de España.

\section{TWO NEW DRAWINGS BY CLAUDIO COELLO IN SPAIN'S BIBLIOTECA NACIONAL}

The authors ascribe to the Madrid painter Claudio Coello (1642-1693) two drawings of a similar theme preserved in the Biblioteca Nacional de España (Spanish National Library), which had until now been considered as anonymous. Key words: Claudio Coello; Drawings; Biblioteca Nacional de España (Spanish National Library).

De los ricos fondos de dibujos anónimos de la Biblioteca Nacional de España creemos que deben ser rescatadas dos hojas espléndidas, las cuales, por sus características formales y su relación con obras seguras del artista, pueden ser atribuidas a Claudio Coello.

Obra de gran belleza es el dibujo titulado Ángeles niños (fig. 1) ${ }^{1}$. Se trata, sin lugar a dudas, de estudios para una Inmaculada Concepción; los grupos de la parte inferior copian de manera bastante fiel modelos utilizados por Carreño de Miranda en algunos de sus cuadros de este asunto, siendo especialmente cercanos a los angelitos que aparecen en la gran Inmaculada de la Hispanic Society of America de Nueva York ${ }^{2}$.

La figura principal del grupo situado en la parte inferior izquierda -el angelito de espaldas, que recorta el perfil de su cabeza sobre el espejo simbólico ${ }^{3}$ - es un modelo que debió de agradar especialmente a Coello, pues este lo introduce en varias versiones de la Inmaculada debidas a su mano, como las conservadas en el Tribunal Supremo ${ }^{4}$ y en la Hermandad del Refugio de San Antonio de los Portugueses ${ }^{5}$, obras de hacia 1685-1690, ambas en Madrid, sin más variación significativa que el cambio de posición de las piernas, lo que le aporta un aire más movido y sinuoso, de acuerdo con el gusto del artista madrileño. Esta solución, adoptada finalmente, aparece ya esbozada en un ligero apunte situado en el ángulo inferior derecho de la propia hoja.

${ }^{1}$ Barcia, 1906: 133, núm. 654, como anónimo español del siglo XVII. Signatura: DIB/15/4/31. Pluma con tinta marrón sobre papel agarbanzado claro. Medidas: 283 x $208 \mathrm{~mm}$.

${ }^{2}$ López Vizcaíno/Carreño, 2007: 268-269.

${ }^{3}$ Es un modelo habitual en algunas Inmaculadas de Carreño, como la de la Catedral Vieja de Vitoria o la del Monasterio de la Encarnación de Madrid, entre otras, y aparece también en el gran lienzo de La Fundación de la Orden de la Trinidad del Louvre. Véase Pérez Sánchez, 1985: 130 y 135.

${ }^{4}$ Sullivan, 1989: 209, P 77. Pérez Sánchez, 1990: 138-139, figs. 6 y 7.

${ }^{5}$ Sullivan, 1989: 208, P 76. 


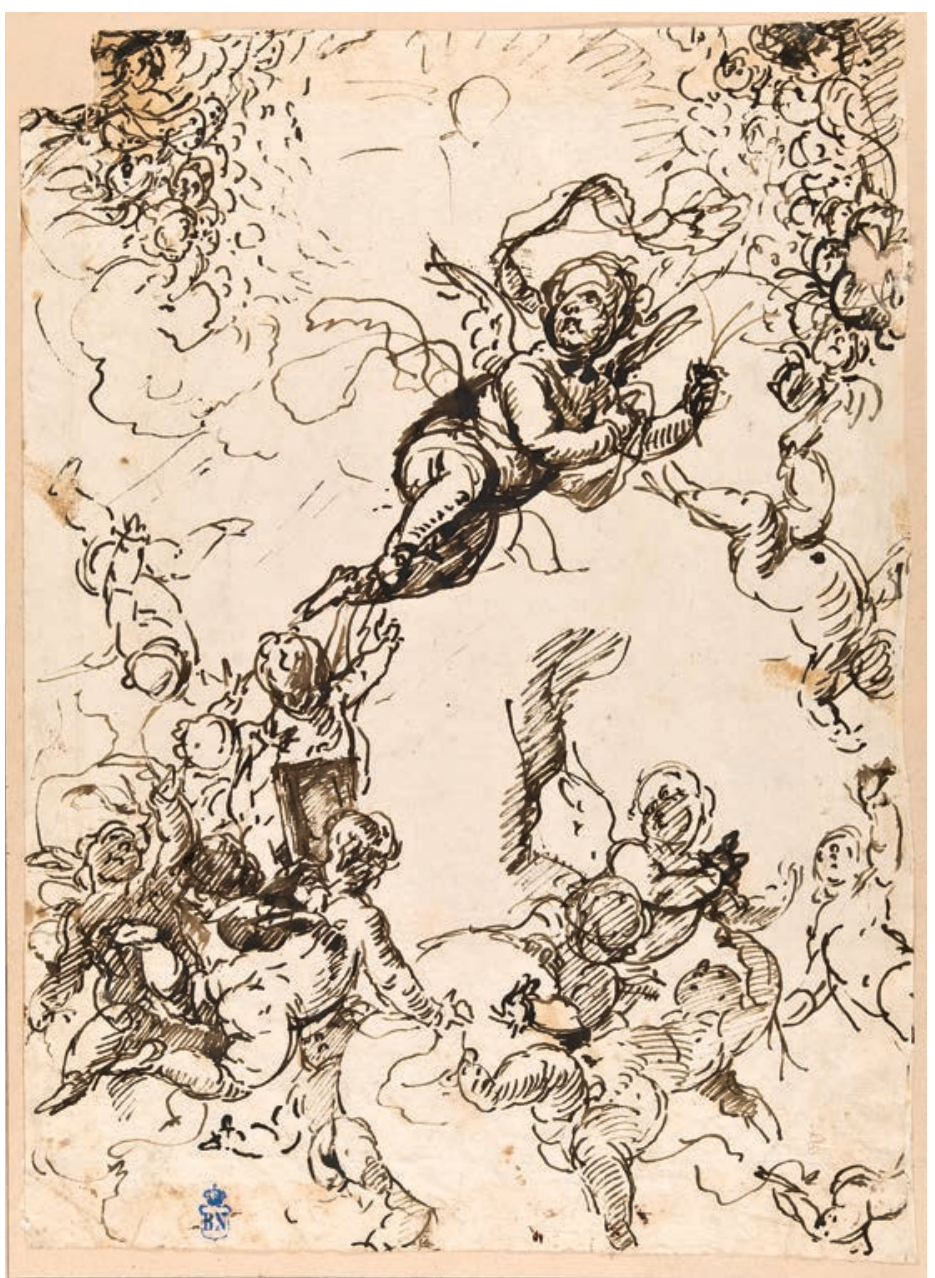

Fig. 1. Claudio Coello. Ángeles niños. Madrid, Biblioteca Nacional de España.

El ángel de mayor tamaño, situado en la parte superior, guarda estrechas semejanzas con uno de los angelitos que aparecen a los pies de la Virgen en el cuadro de la Inmaculada del convento de las MM. Capuchinas de Toledo, obra firmada por Claudio Coello ${ }^{6}$. En este, si bien las piernas se corresponden de forma casi literal con las del dibujo, el pintor parece haber aprovechado el brazo derecho de la figura dibujada, doblado ante el pecho agarrando una cinta o cendal, colocándolo en el mismo lugar como brazo derecho del angelito situado a su lado, que pasa a sostener el ramo de azucenas (fig. 2).

Entre el grupo de angelitos de la zona inferior derecha, merece especial mención el que, de medio cuerpo sobre el perfil del globo terráqueo, inclina su cabeza hacia el ángel de cuerpo entero visto en escorzo bajo él, el cual adopta una torsión característica del pintor $^{7}$ y recuerda una figura

\footnotetext{
${ }^{6}$ Nicolau Castro, 1984: 385, fig. 20. Pérez Sánchez, 1990: 139, fig. 8.

7 A modo de ejemplo, véase la figura masculina cubierta con un manto, a contraluz, detrás del santo, en el San Felipe de la iglesia del convento de Santa Isabel de Madrid (desaparecido), o la de San José en el lienzo de La Virgen y el Niño adorados por San Luis y otros santos del Museo del Prado (Sullivan, 1989: 167-168, P 10 y 171-172, P 15).
}

Arch. esp. arte, LXXXVIII, 351, JULIO-SEPTIEMBRE 2015, 310-314 ISSN: 0004-0428, eISSN: 1988-8511, doi: 10.3989/aearte.2015.20 


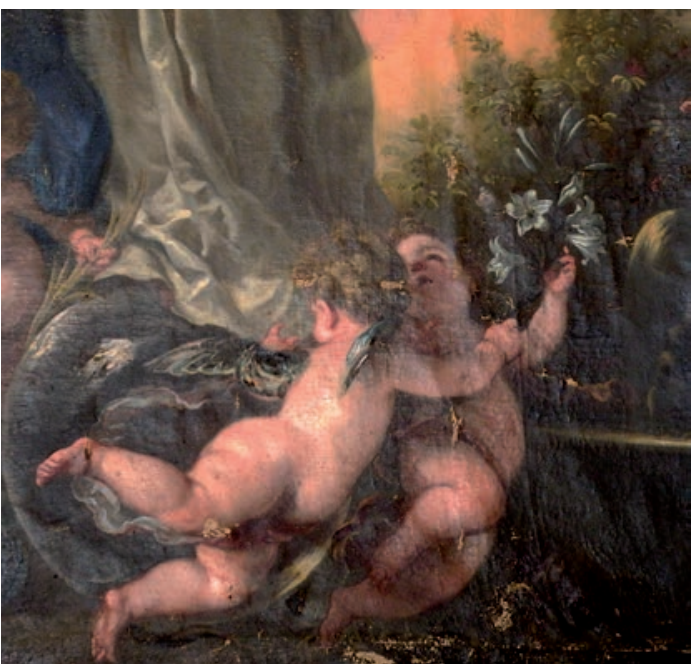

Fig. 2. Claudio Coello. Inmaculada (detalle). Toledo, convento de MM. Capuchinas. Foto: Fundación Las Edades del Hombre.

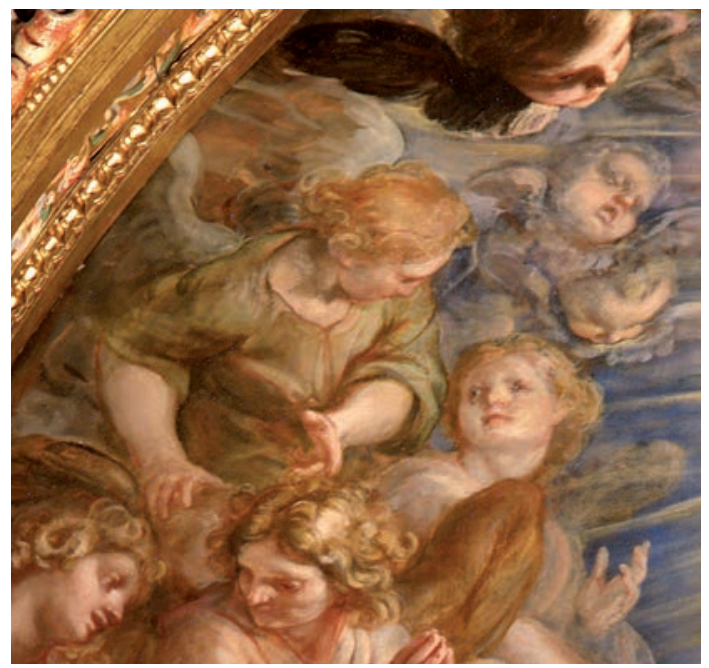

Fig. 3. Claudio Coello. La Anunciación (detalle). Madrid, iglesia del convento de San Plácido. Foto: Juan Carlos Martín Lera, Dirección General de Patrimonio Histórico de la Comunidad de Madrid.

similar que aparece entre la gloria de ángeles, alrededor del Dios Padre, en la Anunciación de la iglesia de San Plácido de Madrid (fig. 3). Los apuntes de la parte superior, una figura circular rodeada de resplandores y una gloria de cabecitas angélicas, si bien sería lógico pensar que fuesen igualmente estudios para una Inmaculada -quizá para la parte alta de la figura, con la cabeza apenas esbozada, como señala Barcia-, creemos que por su forma sugieren más bien la representación de una hostia en un cáliz, al modo de la Alegoría del Sacramento de la Eucaristía que aparece en la puerta del sagrario del retablo mayor de la misma iglesia de San Plácido ${ }^{8}$.

La obra presenta claras semejanzas en el uso de la pluma con otros dibujos de Coello realizados con una técnica similar, como el Estudio para el frontispicio de "El Buen Pastor" de la misma Biblioteca Nacional $1^{9}$, de 1682, o el Techo con Flora del Prado ${ }^{10}$ (h. 1680-1685), especialmente con este último, y es ejemplo bellísimo de la maestría y seguridad del pintor: sin más guía que sus ojos, puesto que no hay rastros de lápiz bajo la tinta, como es usual en este tipo de obras, el artista traza velozmente las formas sobre la hoja en blanco, con agilidad y frescura.

El segundo dibujo es el titulado por Barcia Geniecillos o ángeles niños ${ }^{11}$ (fig. 4). Figuras semejantes aparecen en varias obras pintadas por Claudio Coello. Así, por ejemplo, el angelito situado en zona inferior de la hoja, fuertemente escorzado hacia atrás, del que solo es visible el brazo derecho alzado, es muy parecido al que sobrevuela la escena con una corona de flores en el lienzo de $L a$ Virgen y el Niño adorados por santos y las virtudes teologales del Museo del Prado (fig. 5), o a otra

\footnotetext{
${ }^{8}$ Gutiérrez Pastor, 2003: 136, fig. 13.

9 Barcia, 1906: 66, núm. 319. Signatura: DIB /16/40/7. Preparado a lápiz negro. Pluma con tinta parda sobre papel agarbanzado. Medidas: 242 x $171 \mathrm{~mm}$. Sullivan, 1989: 262, D29.

${ }^{10}$ Número de catálogo: D03811. Preparado a lápiz negro. Pluma con tinta marrón sobre papel agarbanzado. Medidas: 250 x 175 mm. Sullivan, 1989: 268, D41.

${ }^{11}$ Barcia, 1906: 132, núm. 651, como anónimo español del siglo XVII. Signatura: DIB/13/3/4. Sanguina y lápiz negro sobre papel agarbanzado. Medidas: 200 × $256 \mathrm{~mm}$. Catalogado actualmente como anónimo italiano del siglo XVII.
}

Arch. esp. arte, LXXXVIII, 351, JULIO-SEPTIEMBRE 2015, 310-314 ISSN: 0004-0428, eISSN: 1988-8511, doi: 10.3989/aearte.2015.20 


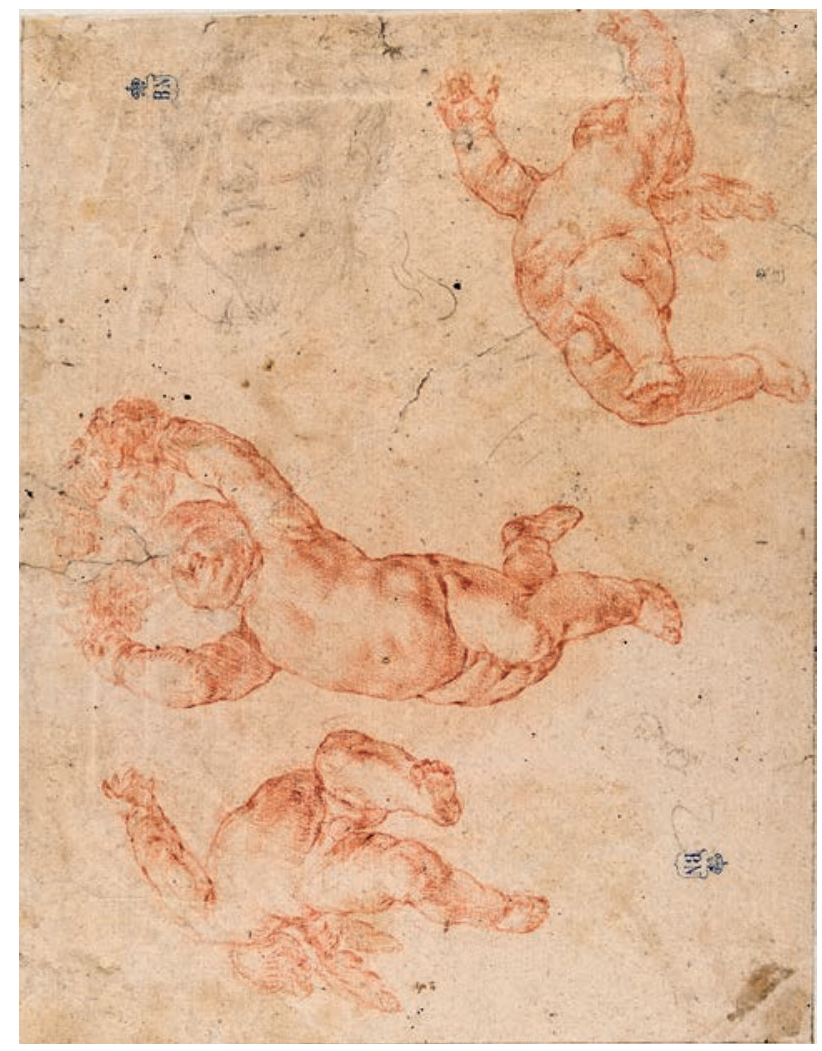

Fig. 4. Claudio Coello. Geniecillos o ángeles niños. Madrid, Biblioteca Nacional de España.

figura similar de La aparición de la Virgen y el Niño a Santo Domingo de Guzmán de la Academia de Bellas Artes de San Fernando de Madrid ${ }^{12}$. Asimismo, el angelito que sostiene la corona de flores en el dibujo recuerda a la figura que porta una corona de rosas en el mismo cuadro de la Academia, y a otra del enorme lienzo del Éxtasis de Santa María Magdalena -el angelito con el flagelo, entre nubes, a los pies de la santa- en la iglesia de Santa María Magdalena de Ciempozuelos (Madrid) ${ }^{13}$.

El modelado es muy apretado y seguro, y sugiere el volumen con extraordinaria maestría. En este sentido, es especialmente notable la figura que porta la corona, cuya ejecución, a base de trazos paralelos muy cortos y unidos, le confiere una solidez casi escultórica. Es la misma forma de sugerir el modelado de la anatomía que aparece en la conocida Academia del Museo Casa de la Moneda de Madrid (fig. 6), cuya impresión de solidez es más acentuada aún en la obra que estudiamos, diferencia que tal vez se deba a que esta sea de fecha algo posterior, quizá de la década de 1670, de acuerdo con la evolución de la pintura de Coello hacia formas de volúmenes más acusados y perfiles más cerrados.

La presencia junto a los angelitos de una cabeza masculina -un religioso sin duda-permite pensar en la posibilidad de que la obra fuese preparatoria para algún cuadro que representase la figura de un santo -tal vez dominico, a juzgar por el tipo de cuello del hábito-, bien con la imagen del santo solo, como el Santo Domingo de Guzmán del Prado ${ }^{14}$, o coronado por ángeles, como el San

\footnotetext{
${ }^{12}$ Sullivan, 1989: 182-183, P 44.

${ }^{13}$ Sullivan, 1989: 201-202, P 69.

${ }^{14}$ Número de catálogo: P 00662. Sullivan, 1989: 206, P73.
} 


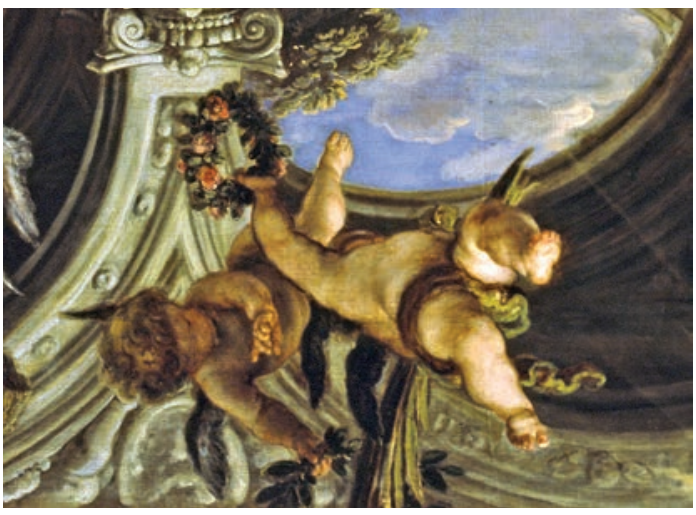

Fig. 5. Claudio Coello. La Virgen y el Niño adorados por santos y las virtudes teologales (detalle invertido). Madrid, Museo Nacional del Prado.

Fig. 6. Claudio Coello. Academia. Madrid, Museo Casa de la Moneda.

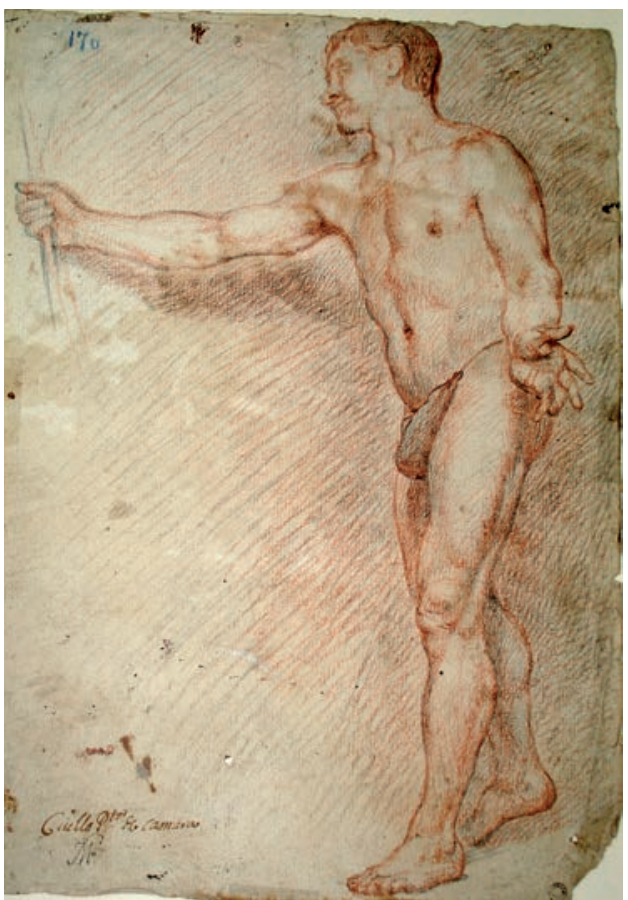

Francisco Javier de la iglesia de Nuestra Señora de la Asunción de Valdemoro (Madrid) ${ }^{15}$, o en alguna composición más compleja, del tipo de La aparición de la Virgen y el Niño a Santo Domingo de Guzmán de la Academia de Bellas Artes de San Fernando de Madrid, ya mencionada, hipótesis que refuerza la propia expresión de arrobo o éxtasis de la cabeza, con los ojos vueltos hacia lo alto.

\section{BIBLIOGRAFÍA}

Barcia, Ángel M. de (1906): Catálogo de la colección de dibujos originales de la Biblioteca Nacional. Madrid: Tipografía de la Revista de Archivos, Bibliotecas y Museos.

Gutiérrez Pastor, Ismael (2003): "Novedades sobre Claudio Coello, con algunas cuestiones iconográficas y compositivas". En: Anuario del Departamento de Historia y Teoría del Arte, vol. XV, Universidad Autónoma de Madrid, pp. 125-145.

López Vizcaíno, Pilar / Carreño, Ángel Mario (2007): Juan Carreño Miranda. Vida y obra. Oviedo.

Nicolau Castro, Juan (1984): "Inmaculadas firmadas por C. Coello y M. de Zayas en Toledo". En: Archivo Español de Arte, tomo LVII, núm. 228, pp. 383-386.

Pérez Sánchez, Alfonso Emilio (1985): Juan Carreño de Miranda (1614-1685). Ayuntamiento de Avilés. Pérez Sánchez, Alfonso Emilio (1990): "En torno a Claudio Coello". En: Archivo Español de Arte, tomo LXIII, núm. 250, pp. 129-155.

Sullivan, Edward J. (1989): Claudio Coello y la pintura barroca madrileña. Madrid: Editorial Nerea.

Fecha de recepción: 06-VI-2014

Fecha de aceptación: 22-XII-2014

${ }^{15}$ Sullivan, 1989: 198, P 64.

Arch. esp. arte, LXXXVIII, 351, JULIO-SEPTIEMBRE 2015, 310-314 ISSN: 0004-0428, eISSN: 1988-8511, doi: 10.3989/aearte.2015.20 